\title{
Optimized Yellow Dwarf Virus Multiplex PCR Assay Reveals a Common Occurrence of Barley yellow dwarf virus-PAS in Kansas Winter Wheat
}

\begin{abstract}
Alma G. Laney, Kansas State University, Department of Plant Pathology, Manhattan, 66506, and North Carolina State University, Department of Entomology and Plant Pathology, Raleigh, 27606 (current); Rodolfo Acosta-Leal, Kansas State University, Department of Plant Pathology, Manhattan, 66506, and Murray State College, Tishomingo, OK 73460 (current); and Dorith Rotenberg, ${ }^{\dagger}$ Kansas State University, Department of Plant Pathology, Manhattan, 66506, and North Carolina State University, Department of Entomology and Plant Pathology, Raleigh, 27606 (current)
\end{abstract}

Accepted for publication 15 December 2017.

\section{Abstract}

Barley yellow dwarf is an aphid-transmitted virus disease caused by yellow dwarf virus (YDV) species in the family Luteoviridae. Previous partial sequencing efforts conducted in Kansas revealed that Barley yellow dwarf virus-PAS (PAS) occurs in winter wheat fields, and currently available YDV multiplex reverse-transcription PCR (RT-PCR) assays do not detect this species. To enable precise determination of YDV species for research, disease diagnostic, and plant breeding programs, this study enhanced and validated the utility of a multiplex RT-PCR protocol to discriminate six YDV species, including PAS, in archived and fresh field samples. From a representative subset of samples collected from commercial and variety trial locations across nine wheat growing regions of Kansas, PAS and Barley yellow dwarf virus-PAV (PAV) were equally prevalent in single or mixed infections, and other YDVs occurred in mixed infections with PAS and/or PAV in low numbers. The optimized multiplex assay provided robust and specific detection of YDVs and showed promise as a diagnostic tool for determining species occurrence and composition of YDVs in an intensive wheat cropping region of the United States.
Barley yellow dwarf (BYD) is a complex and economically important disease caused by multiple virus species collectively known as yellow dwarf viruses (YDVs). YDVs infect over 100 species of Poaceae (D'Arcy and Domier 2000), including agronomic grasses (wheat, barley, oats, maize, and rice) and wild grasses such as big bluestem and switchgrass (Garrett et al. 2004). Species of YDV are discriminated by differential responses to antisera, differences in host range, and variations greater than $10 \%$ in the amino acid sequence of any one gene product (Domier 2012). YDVs belong to the family Luteoviridae and are classified in the genera Luteovirus and Polerovirus. The YDVs in the genus Luteovirus include Barley yellow dwarf virus (BYDV)-PAV (hereafter PAV; Miller et al. 1988), BYDV-PAS (hereafter PAS; Chay et al. 1996), BYDVMAV (hereafter MAV; Ueng et al. 1992), BYDV-KerII and BYDV-KerIII (Svanella-Dumas et al. 2013), BYDV-GAV (hereafter GAV; Jin et al. 2004), BYDV-PAV-CN (Liu et al. 2007), and BYDV-SGV (hereafter SGV; Rochow and Muller 1971). Members of the genus Polerovirus include Cereal yellow dwarf virus (CYDV)-RPV (hereafter RPV; Vincent et al. 1991), CYDV-RPS (Mayo 2002), Wheat yellow dwarf virus-GPV (hereafter GPV; Zhang et al. 2009), and Maize yellow dwarf virus-RMV (hereafter RMV; Krueger et al. 2013). A variant of RMV identified in

${ }^{\dagger}$ Corresponding author: Dorith Rotenberg; E-mail: drotenb@ncsu.edu

*The $\boldsymbol{e}$-Xtra logo stands for "electronic extra" and indicates that one supplementary table is published online.

Funding was provided by Kansas Wheat Commission. Heartland Plant Innovations, Inc., funded the Kansas wheat survey that provided the archived field isolates.

(C) 2018 The American Phytopathological Society
Montana was determined to have a larger vector and host range, including wheat, than the type isolate that primarily infects corn (hereafter RMV-MT; Geske et al. 1996).

Developing reliable and accurate methods for distinguishing YDVs has been a challenge with the emergence of new species and strains. Two multiplex reverse-transcription PCR (RT-PCR) protocols are currently available for YDV detection in plant tissue and have been used extensively by researchers (Deb and Anderson 2008; Malmstrom and Shu 2004). Malmstrom and Shu (2004) designed an assay to distinguish four YDV species: PAV, MAV, SGV, and RPV, and the method was tailored to enrich for YDV sequences in the cDNA synthesis step by targeting a highly conserved region in the coat protein $(\mathrm{CP})$ open reading frame (ORF). Deb and Anderson (2008) developed a multiplex RT-PCR protocol to detect an additional species, RMV, along with other common wheat viruses to provide discrimination of eight wheat viruses. These methods were further refined to discriminate YDVs by adding a restriction digestion step of CP amplicons to distinguish MAV, PAS, and PAV (Kundu et al. 2009). However, there are no published multiplex RT-PCR protocols available to differentiate between PAV and PAS directly. In addition, the accumulation of publically available full-length YDV genome sequences in the past 10 years offers the opportunity to optimize discrimination of YDV species and genetic variants in wheat.

Recent work in Kansas highlights the need for an updated YDV multiplex detection protocol that includes PAS. In Kansas, BYD is a chronic disease of winter wheat, causing an estimated $1 \%$ in annual grain yield losses (Appel et al. 2015), and during epidemic years it can lead to an estimated $\$ 78.1$ million in losses (based on data from the Kansas Department of Agriculture 2013). A 2-year sample-intensive statewide winter wheat survey was conducted to 
determine, in part, the occurrence of six economically significant virus species, including two YDVs, in Kansas fields (Rotenberg et al. 2016). Using enzyme-linked immunosorbent assays (ELISAs) with commercially available polyclonal antibodies to viral CPs, the study revealed that PAV was the most prevalent and widely distributed virus species across Kansas in those 2 years. The survey sampled equivalent numbers of symptomatic and asymptomatic tillers, of which one-third were ELISA-positive for PAV; conversely, $31 \%$ were symptomatic yet ELISA-negative for all six species in the panel, an indication of the detection limit of ELISA protocols or the occurrence of unaccounted virus species or both. Partial genome sequencing of a subset of archived PAV-positive isolates from the survey revealed the presence of PAS (Acosta-Leal et al. 2013, APS regional meeting abstract). Apparently, the use of the PAV antibody did not differentiate between PAV and PAS in the survey, a phenomenon that has been previously described for ELISA-based detection of closely related YDVs, such as PAS and PAV (Chay et al. 1996). The objectives of this study were to (i) sequence genomes of two field isolates of PAS and one of PAV to enable primer design to capture Kansas haplotypes, (ii) modify and optimize the existing multiplex assays to discriminate PAV, PAS, MAV, SGV, RPV, and RMV-MT; and (iii) validate the multiplex assay using archived and fresh leaf samples from Kansas wheat.

\section{Variants of PAS and PAV Isolates Occur in Kansas}

Two single-isolate leaf-tissue samples $(100 \mathrm{mg}$ ) of PAS (AcostaLeal et. al. 2013, meeting abstract) stored at $-80^{\circ} \mathrm{C}$ and one sample of PAV from the field survey (Rotenberg et al. 2016) were selected for genome sequencing. The tissue was pulverized using the Fast-Prep 24 (MP Biomedical, Solon, OH) followed by RNA extraction through the RNeasy Plant Mini kit (Qiagen, Germantown, MD) using the manufacturer's specifications. Using 1,000 ng of total RNA, cDNA was transcribed using Transcriptor (Roche, Indianapolis, IN) and a reverse primer designed to be specific for the $3^{\prime}$ end of PAS (5'-GGGATGCCGGGCTTCTCTTTCG-3') or PAV (5'-GGGTTGCCGAACTGCTCTTTCG-3') based on consensus sequences from multiple alignments of full-length PAS or PAV genome sequences available in the National Center for Biotechnology Information (NCBI) GenBank.

Internal primers were designed to amplify each cDNA sequence in four overlapping fragments with at least $200 \mathrm{bp}$ overlap to allow for assembly. The fragments were then amplified using PrimeSTAR GXL (Takara Bio USA, Mountain View, CA), cloned using the Zero Blunt TOPO kit (Thermo Fisher Scientific, Waltham, MA), and transformed into TOP10 cells (Thermo Fisher Scientific) using kanamycin selection. Colonies growing on Luria broth agar + kanamycin were screened by colony PCR, and plasmids were isolated using the Plasmid Mini kit (Qiagen, Germantown, MD), digested with EcoRI to verify expected insert size, and sent to Genewiz (South Plainfield, NJ) for sequencing. At least a $3 \times$ genome coverage was obtained for each isolate, and sequences were assembled in CAP3 (Huang and Madan 1999).

Assembled sequences were confirmed to be PAS or PAV using NCBI BLAST (Altschul et al. 1990) and ORFs were predicted using NCBI ORF Finder (Rombel et al. 2002). Sequencing to a $3 \times$ genome coverage revealed contiguous lengths of 5,641, 5,671, and 5,675 nt for KS PAV, KS PAS-1, and KS PAS-2, respectively. The KS PAS and KS PAV genomes encoded the six ORFs typical of the luteovirus genomes (Domier 2012).

Sequence analysis of the Kansas PAS and PAV single-tiller winter wheat isolates revealed some level of genetic variation from publicly available reference sequences. Using Clustal Omega (Sievers et al. 2014), pairwise percent identities were determined for each ORF (nucleotide) and gene product (amino acid) sequence compared with NCBI GenBank reference sequences (PAS [NC_002160.1] and PAV [NC_004750.1]) and two additional PAV accessions: PAV-064 (EF521850.1) and PAV-109 (EF521828.1). The two ORFs encoding for the replication machinery (RDRP), ORFs 1 and 2, as well as the CP and movement protein (MP) encoded by ORFs 3 and 4, shared typically high degrees of identity to their respective reference sequences (Table 1). Surprisingly, the two PAVdesignated GenBank accessions (PAV-064 and PAV-109) had greater amino acid identities with the two KS PAS sequences (96 to $100 \%$ ) than with the KS PAV sequence (78 to $88 \%$ ) for RDRP, CP, and MP, indicating that the two accessions may be classified more accurately as PAS isolates. The predicted ORFs comprising the $3^{\prime}$ proximal region, that is, the read-through protein (RTP) (ORF5) and P6 (ORF6), were more variable, with P6 having 43 to 55\% amino acid identity to their respective reference sequence. One of the criteria for demarcation of distinct luteovirus species is one or more ORFs sharing less than $90 \%$ amino acid identity. All three Kansas isolates meet this criterion with P6, and the RTP is at or around this cutoff with 90 to $93 \%$ amino acid identity. However, other criteria including transmission properties, host range, and vector range distinguish luteovirus species. As such, studies are underway to fill in these knowledge gaps for the KS PAS variants reported in this study.

\section{An Optimized Multiplex RT-PCR Was Designed to Ensure Species Specificity and to Capture the Genetic Variation in Kansas Isolates}

Primer design. The optimized set of RT-PCR primers used in this study is listed in Table 2, and their intended genome alignment positions are illustrated in Figure 1. As a first step to PCR optimization, published primer sequences retrieved from two protocols designed for ORF3 (CP) for multiplex identification of YDV species (Deb and Anderson 2008; Malmstrom and Shu 2004) were subjected to BLASTn alignments with all GenBank YDV sequences available as of January 2015 to determine sequence specificity in silico. We found evidence of nonspecific priming in silico and in practice (data not shown) for most of the previously published primers. The ShuF primer was originally designed by Malmstrom and Shu (2004) to target multiple YDV species, and at least $90 \%$ of the primer matched the GenBank sequences without any mismatches in the $3^{\prime}$ half of the primer; many accessions were a $100 \%$ match for this primer, and use of this primer in multiplex resulted in several additional bands (data not shown). The reverse primer for SGV (SGVR), the forward primer for RPV (RPVL) (Malmstrom and Shu 2004), and the PAV primer pair (PAVL and PAVR; Deb and Anderson 2008) appeared to be species specific and were therefore retained in the final primer set in the present study. To ensure specificity, new primers were designed for ORF3 (CP) of RPV (reverse primer), SGV (forward primer), and RMVMT and for the ORF5 (RTP) of MAV using all publicly available sequences for each species, and in a manner described previously (Dieffenbach et al. 1993; Wu et al. 1989), whereby primers that had one to two mismatches between species in the last four bases of the $3^{\prime}$ end of the primer were selected for testing. Mismatches between the primer and template at the terminal $3^{\prime}$ end of the primer prevent the Taq polymerase from extending beyond the primer $3^{\prime}$ end and ensure target specificity for the primer set.

For PAS, early attempts at designing a primer set in ORF5 (RTP) predicted species specificity in silico; however, this primer set failed to capture the diversity of PAS isolates that occur in Kansas. We discovered this to be the case because the number of archived field survey samples determined to be PAS positive using the ORF5 primers was grossly underestimated compared with the number of PAS positives determined using HpaII digestion of the YanR/ShuF 
CP/MP (ORF3/ORF4) amplicon for discriminating PAS from PAV (Kundu et al. 2009). Using the newly generated PAS-1 and PAS-2 genome sequences and the PAS reference sequence accession (NC_002160.1), a new primer set was designed in ORF2 (RDRP). This new primer set in the multiplex was specific to PAS, generating results that were consistent with the HpaII-digestion confirmation step (Fig. 2).

An in silico analysis was performed on the newly designed and retained multiplex primers (14 primer sequences in total) to demonstrate primer specificity to the intended YDV species. Using a collection of 92 YDV sequences composed of complete genomes of GAV, GPV, MAV, PAS, PAV, PAV-CN, RMV, and RPV, partial sequences of SGV and RMV-MT, and the single sequence of the wheat ubiquitin reference gene, BLASTn for short oligos was performed to evaluate the likelihood of nonspecific priming of off-target YDVs. Supplementary Table 1 depicts the features used to evaluate specificity. Overall, in the off-target hits, mismatches occurred not only along the length of the primer but also in the four bases at the 3 '-terminal end, and in the few cases in which a primer sequence had a near or complete match (e.g., RPVL) to an off-target template sequence, its primer partner (RPVR) had a poor match to that template or the primer pair alignments were in incorrect orientation for PCR.

For the cDNA synthesis step, the published general YDV primer YanR (Malmstrom and Shu 2004) was confirmed to match all available YDV sequences (luteo- and poleroviruses) in the ORF3 (CP) region, thus enriching for YDV templates. For MAV, speciesspecific PCR priming sites could not be found in ORF3; thus, we extended primer design to ORF5 (RTP) and designed a second cDNA synthesis primer for this region for MAV accordingly that also matched other luteoviruses, including PAS and PAV.

To provide some level of confidence in reporting YDV "negatives", internal reference primers (cDNA and PCR) were included in the multiplex assay for detection of wheat ubiquitin RNA for a gene previously reported as a potential internal reference gene in wheat (UniGene Cluster Ta50503, Paolacci et al. 2009). Including the ubiquitin reference primers, all primer sets in multiplex produced amplicon sizes that differed by at least 25 bp (Fig. 2).

Test of RT-PCR in mock single and mixed infections. Singleinfection positive controls for MAV, PAS, SGV, RPV, and RMVMT in Coast Black oat provided by S. Gray (USDA-ARS, Cornell University) and the KS PAS-2 and KS PAV isolates sequenced in this study were used as cDNA templates in equal parts singly or in three types of artificial mixtures based on natural YDV occurrence: KS “major” group (PAV, PAS, and RPV), KS “minor” group (MAV, SGV, and RMV-MT), and "full" group combining templates of all six species. Ubiquitin primers were included in all reactions. Tissue $(100 \mathrm{mg}$ ) was flash-frozen in liquid nitrogen, and total RNA was isolated using the methods described above for the

TABLE 1

Nucleotide and amino acid conservation (\% identity) between the genome-sequenced Kansas (KS) Barley yellow dwarf virus-PAS (PAS-1 and PAS-2) and -PAV field isolates and representative publically available sequences of the same species for the six genes ${ }^{a}$

\begin{tabular}{|c|c|c|c|c|c|c|c|}
\hline Sequence & KS PAS-1 & KS PAS-2 & KS PAV & PAV & PAS & PAV 064 & PAV 109 \\
\hline \multicolumn{8}{|l|}{$\mathrm{P} 1$} \\
\hline KS PAS-1 & N/A & $94 \%(96 \%)$ & $77 \%(81 \%)$ & $76 \%(79 \%)$ & $96 \%(97 \%)$ & $96 \%(97 \%)$ & $94 \%(96 \%)$ \\
\hline KS PAS-2 & $94 \%(96 \%)$ & N/A & $76 \%(79 \%)$ & $75 \%(80 \%)$ & $94 \%(96 \%)$ & $94 \%(97 \%)$ & $99 \%(98 \%)$ \\
\hline KS PAV & $77 \%(81 \%)$ & $76 \%(79 \%)$ & N/A & $93 \%(98 \%)$ & $77 \%(80 \%)$ & $76 \%(80 \%)$ & $76 \%(78 \%)$ \\
\hline \multicolumn{8}{|l|}{$\mathrm{P} 1 / \mathrm{P} 2$ fusion } \\
\hline KS PAS-1 & N/A & $93 \%(96 \%)$ & $80 \%(85 \%)$ & $80 \%(85 \%)$ & $97 \%(98 \%)$ & $97 \%(97 \%)$ & $93 \%(96 \%)$ \\
\hline KS PAS-2 & $93 \%(96 \%)$ & N/A & $79 \%(85 \%)$ & $79 \%(85 \%)$ & $93 \%(96 \%)$ & $93 \%(96 \%)$ & $99 \%(99 \%)$ \\
\hline KS PAV & $80 \%(85 \%)$ & $79 \%(85 \%)$ & N/A & $96 \%(99 \%)$ & $80 \%(85 \%)$ & $80 \%(85 \%)$ & $79 \%(84 \%)$ \\
\hline \multicolumn{8}{|l|}{$\mathrm{CP}$} \\
\hline KS PAS-1 & N/A & $96 \%(97 \%)$ & $90 \%(88 \%)$ & $90 \%(87 \%)$ & $99 \%(98 \%)$ & $99 \%(99 \%)$ & $96 \%(97 \%)$ \\
\hline KS PAS-2 & $96 \%(97 \%)$ & N/A & $90 \%(87 \%)$ & $90 \%(86 \%)$ & $96 \%(95 \%)$ & $97 \%(97 \%)$ & $99 \%(100 \%)$ \\
\hline KS PAV & $90 \%(88 \%)$ & $90 \%(87 \%)$ & N/A & $95 \%(97 \%)$ & $90 \%(87 \%)$ & $90 \%(88 \%)$ & $90 \%(87 \%)$ \\
\hline \multicolumn{8}{|l|}{ MP } \\
\hline KS PAS-1 & N/A & $98 \%(97 \%)$ & $92 \%(90 \%)$ & $91 \%(88 \%)$ & $99 \%(98 \%)$ & $100 \%(99 \%)$ & $98 \%(97 \%)$ \\
\hline KS PAS-2 & $98 \%(97 \%)$ & N/A & $92 \%(90 \%)$ & $91 \%(89 \%)$ & $97 \%(98 \%)$ & $98 \%(98 \%)$ & $100 \%(100 \%)$ \\
\hline KS PAV & $92 \%(90 \%)$ & $92 \%(90 \%)$ & N/A & $94 \%(87 \%)$ & $92 \%(90 \%)$ & $92 \%(90 \%)$ & $92 \%(90 \%)$ \\
\hline \multicolumn{8}{|l|}{ RTP } \\
\hline KS PAS-1 & N/A & $91 \%(94 \%)$ & $84 \%(88 \%)$ & $87 \%(90 \%)$ & $90 \%(93 \%)$ & $89 \%(92 \%)$ & $91 \%(94 \%)$ \\
\hline KS PAS-2 & $91 \%(94 \%)$ & N/A & $85 \%(88 \%)$ & $88 \%(90 \%)$ & $87 \%(90 \%)$ & $86 \%(89 \%)$ & $99 \%(99 \%)$ \\
\hline KS PAV & $84 \%(88 \%)$ & $85 \%(88 \%)$ & N/A & $86 \%(90 \%)$ & $91 \%(93 \%)$ & $89 \%(92 \%)$ & $85 \%(88 \%)$ \\
\hline \multicolumn{8}{|l|}{ P6 } \\
\hline KS PAS-1 & N/A & $90 \%(76 \%)$ & $58 \%(44 \%)$ & $70 \%(68 \%)$ & $58 \%(55 \%)$ & $57 \%(55 \%)$ & $93 \%(88 \%)$ \\
\hline KS PAS-2 & $90 \%(76 \%)$ & N/A & $55 \%(58 \%)$ & $69 \%(59 \%)$ & $56 \%(47 \%)$ & $56 \%(47 \%)$ & $92 \%(85 \%)$ \\
\hline KS PAV & $58 \%(44 \%)$ & $52 \%(38 \%)$ & N/A & $57 \%(43 \%)$ & $60 \%(46 \%)$ & $59 \%(46 \%)$ & $57 \%(44 \%)$ \\
\hline
\end{tabular}

a Percent identity: nucleotide (amino acid in parentheses). P1 = $39 \mathrm{kDa}$ helicase encoded by ORF 1; P1/P2 fusion = 99 kDa RNA-dependent RNApolymerase expressed as a fusion protein of ORF1 and ORF 2 by a -1 ribosomal frameshift; $\mathrm{CP}=22 \mathrm{kDa}$ coat protein encoded by ORF 3 ; MP $=17 \mathrm{kDa}$ movement protein encoded by ORF 4; RTP $=72 \mathrm{kDa}$ minor coat protein required for aphid transmission expressed as a read-through of ORF 3 and ORF 5 by stop-codon suppression; P6 $=6 \mathrm{kDa}$ protein of unknown function in PAV or PAS; KS-PAS-1 and KS-PAS-2 $=$ single-tiller isolates from hard red winter wheat in Kansas in 2011 and 2012, respectively; KS-PAV = single-tiller isolate from hard red winter wheat in Kansas in 2011; PAV = PAV reference sequence, NC_004750.1; PAS = PAS reference sequence, NC_002160.1; PAV 064 = PAV isolate 064 isolated from oat in Alaska, EF521850.1; and PAV 109 = PAV isolate 109 isolated from barley in Iowa, EF521828.1. N/A = not applicable. 
obtaining RNA for YDV genome sequencing. cDNA was synthesized using 1,000 ng of RNA using the Verso cDNA kit (Thermo Fisher Scientific) per the manufacturer's recommendations with the use of the gene-specific cDNA primers $(0.5 \mu \mathrm{M}$ per primer), and the final cDNA template for PCR was diluted fourfold for use in the multiplex format. The PCR reactions were performed with the GoTaq Flexi kit (Promega, Madison, WI) with optimized primer concentrations in the reaction mixtures as follows: $0.3 \mu \mathrm{M}$

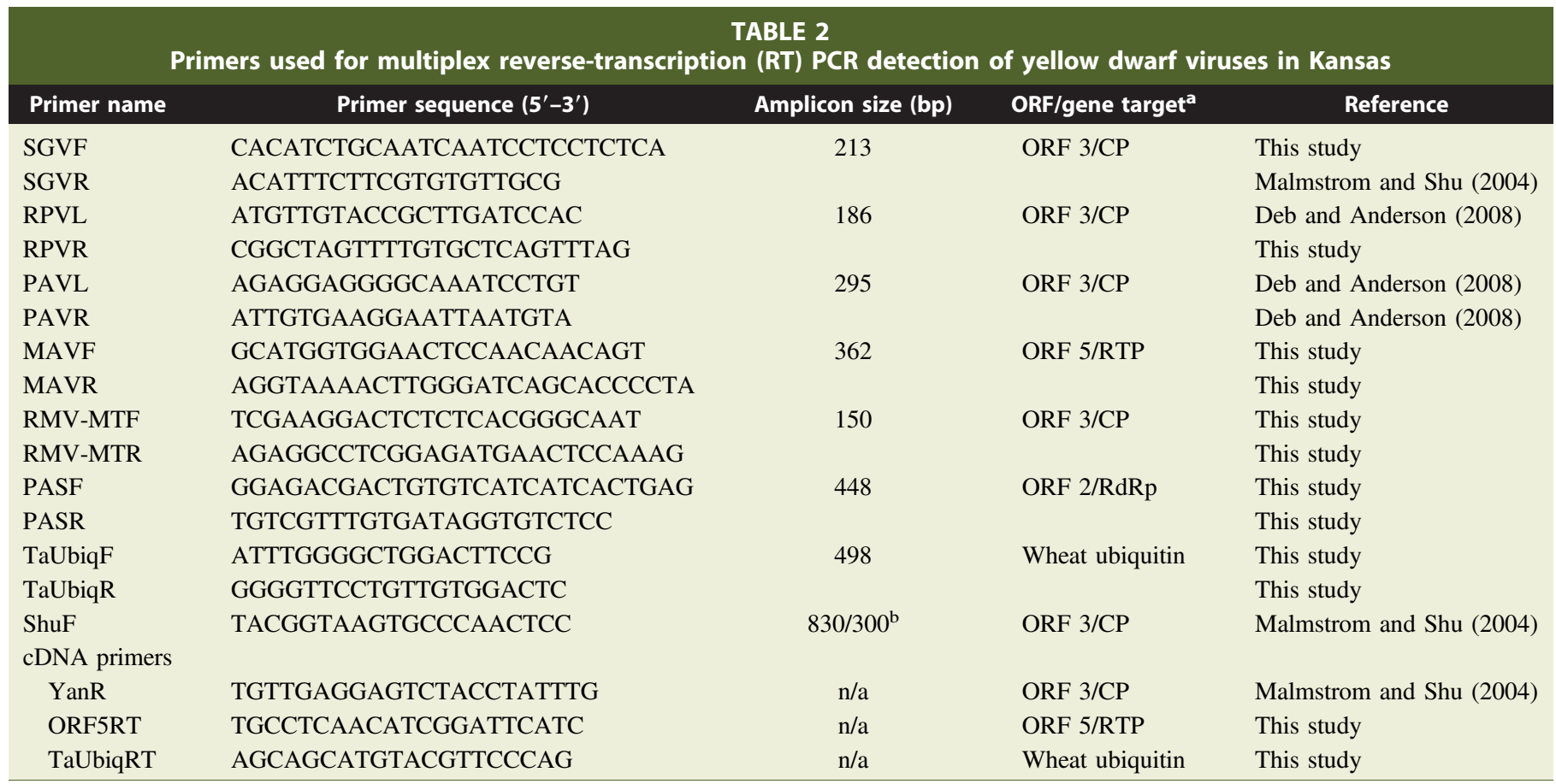

${ }^{\mathrm{a}} \mathrm{ORF}=$ open reading frame; $\mathrm{CP}=$ coat protein; $\mathrm{RTP}=$ read-through domain protein; $\mathrm{RdRp}=\mathrm{RNA}$-dependent $\mathrm{RNA}$ polymerase; and wheat ubiquitin gene $=$ UniGene Cluster Ta50503 (Paolacci et al. 2009).

$\mathrm{b} \sim 830 \mathrm{bp}$ for luteoviruses and $300 \mathrm{bp}$ for poleroviruses when ShuF paired with reverse primer YanR; amplicon used in diagnostic digests to confirm species identification by RT-PCR.

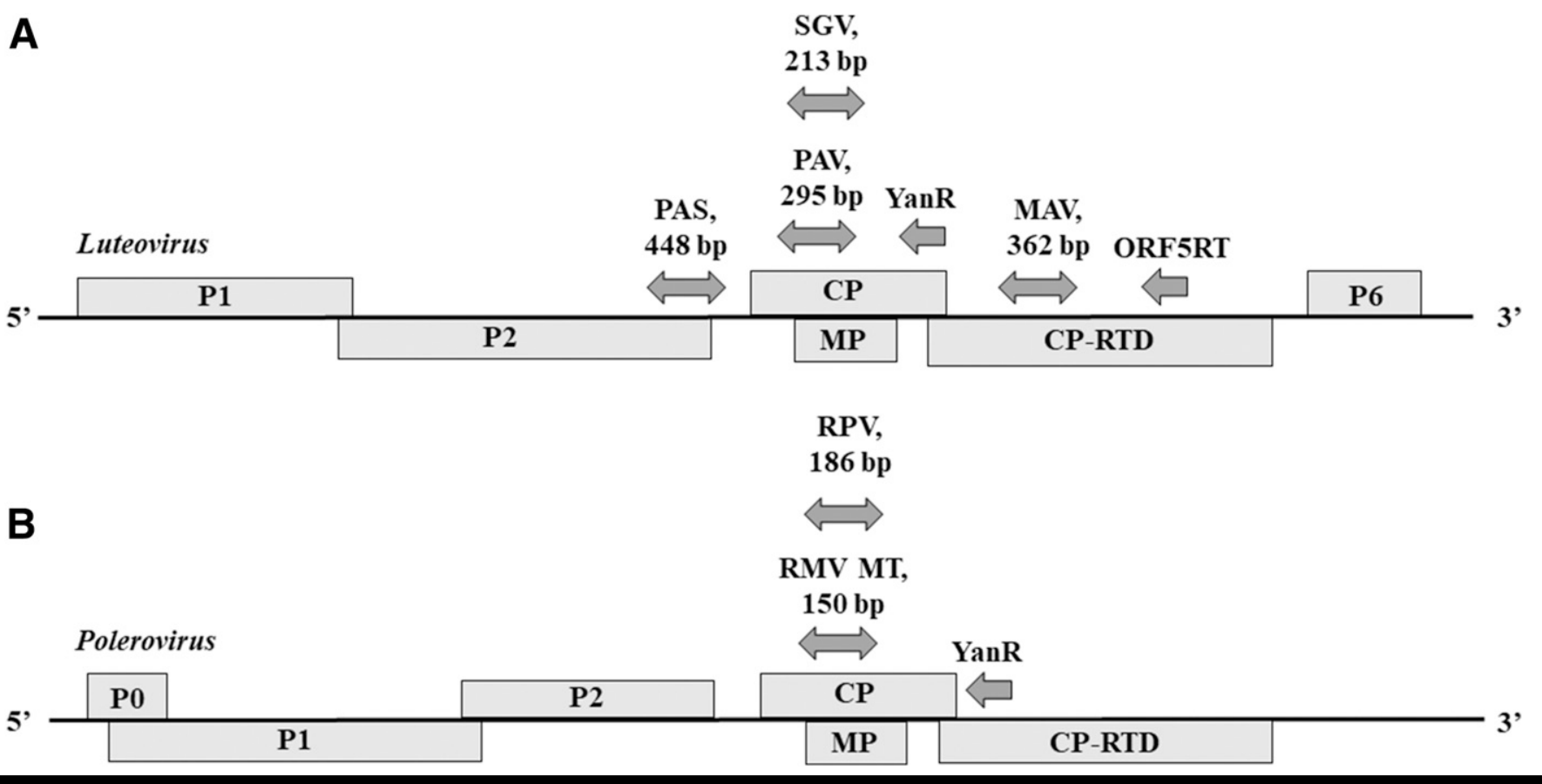

FIGURE 1

Genome regions (double arrows) targeted by multiplex detection primers: A, for luteoviruses: Barley yellow dwarf virus (BYDV)-MAV (MAV), BYDV-PAS (PAS), BYDV-PAV (PAV), and BYDV-SGV (SGV); and B, for poleroviruses: Cereal yellow dwarf virus-RPV (RPV) and a Montana variant of Maize yellow dwarf virus (RMV MT). Single arrows indicate CDNA synthesis reverse primer locations. 


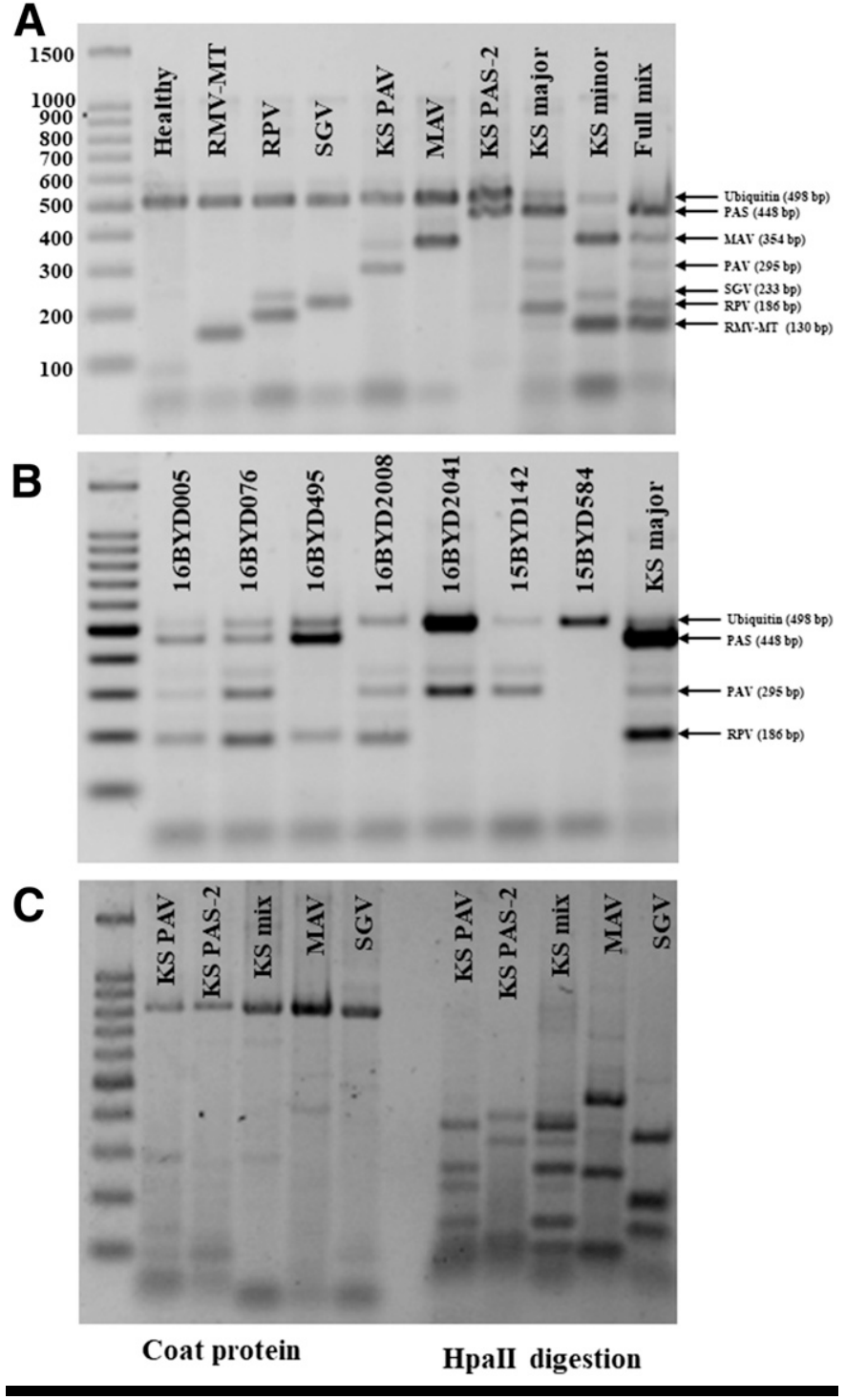

FIGURE 2

A, Multiplex reverse-transcription PCR detection of six yellow dwarf virus (YDV) species in single-isolate positive controls and mock mixtures. From left to right: 100-bp ladder (Promega, Madison, WI); healthy hard red winter wheat cultivar Art (ubiquitin amplicon, 498 bp); Maize yellow dwarf virus-RMV-MT (RMV-MT) (130 bp); Cereal yellow dwarf virus-RPV (RPV) (186 bp); Barley yellow dwarf virus (BYDV)SGV (SGV) (233 bp); BYDV-PAV isolate from Kansas sequenced for this study (KS PAV; 295 bp); BYDV-MAV (MAV) (354 bp); BYDV PAS-2 isolate from Kansas sequenced for this study (KS PAS-2; $448 \mathrm{bp}$ ); mock mixed infection of the most common Kansas YDV isolates (KS major: RPV, KS PAV, and KS PAS-2); less common Kansas YDV isolates (KS minor: RMV-MT, SGV, and MAV); and full mix of all six YDVs (RMV-MT, RPV, SGV, KS PAV, MAV, and KS PAS-2). B, Detection of YDVs in fresh field samples. From left to right: lane $1=100$-bp ladder; lanes 2 to $8=$ seven single tiller leaf samples collected from two breeding nurseries in Kansas; and lane $9=$ the KS major artificial mixture. All lanes show amplification of the wheat ubiquitin internal reference RNA. C, Confirmation of multiplex results by amplification of the coat protein region ( $~ 830$ bp) (lanes 2 to 6 ) followed by amplicon restriction digest (lanes 8 to 12). Lane $1=100$-bp ladder; lanes 2 and $3=$ KS PAV and KS PAS-2 isolates of this study; lane $4=$ a mixed infection of KS PAS-2 and KS PAV from a Kansas greenhouse culture; lanes 5 and $6=$ positive singleinfection controls of MAV and SGV; and lane $7=$ empty. Lanes 8 to 12 are amplicon digestion products: $\mathrm{KS}$ PAV $=369,259,132$, and 71 bp; KS PAS- $2=391$, 325,71 , and $44 \mathrm{bp}$ ) in single (lanes 8 and 9, respectively) and mixed infection (lane 10); MAV $=447,246,71$, and $64 \mathrm{bp}$; and SGV $=336,187,186,115$, and $6 \mathrm{bp}$ (predicted). (ubiquitin), $0.2 \mu \mathrm{M}$ (PAV and SGV), $0.1 \mu \mathrm{M}$ (PAS and RMV-MT), and $0.05 \mu \mathrm{M}$ (MAV and RPV). The other PCR reaction components were $1 \times$ Green GoTaq Flexi buffer, $4 \mathrm{mmol} \mathrm{MgCl}_{2}, 1.5$ units of GoTaq Felxi DNA polymerase, $0.8 \mathrm{mM}$ dNTP mix $(0.2 \mathrm{mM}$ each dNTP), $4 \mu$ l diluted cDNA template, and diethyl pyrocarbonatetreated water to a final volume of $50 \mu \mathrm{l}$. The PCR protocol was $95^{\circ} \mathrm{C}$ for $10 \mathrm{~min}$ followed by 35 cycles of $95^{\circ} \mathrm{C}$ for $30 \mathrm{~s}, 56^{\circ} \mathrm{C}$ for $45 \mathrm{~s}$, and $72^{\circ} \mathrm{C}$ for $1 \mathrm{~min}$, followed by incubation at $72^{\circ} \mathrm{C}$ for $5 \mathrm{~min}$ using a Bio-Rad S1000 thermocycler (Hercules, CA). The amplicons were resolved on a $2 \%$ Tris-borate-EDTA agarose gel with the Promega $100 \mathrm{bp}$ ladder diluted threefold and run at 75 volts for approximately $80 \mathrm{~min}$ and were stained with GelRed (Biotium, Fremont, CA) for $30 \mathrm{~min}$ after electrophoresis according to the manufacturer's recommendations followed by a 5-min destaining in distilled $\mathrm{H}_{2} \mathrm{O}$.

Prominent single amplicons of the expected size for each YDV species target were confirmed in single and multiplex format for the major, minor, and full set of YDVs (Fig. 2A). Most often, five of the six expected amplicons in the complete set of YDVs were easily discernible, with the sixth amplicon migrating closely with the ubiquitin reference (Fig. 2A, final lane). Additional faint bands were occasionally resolved in the multiplex reactions (Fig. 2A and $\mathrm{B}$ ), and upon sequencing it was determined that they occurred because of excess carryover of YanR primer in the PCR step acting with particular forward primers to result in amplification of longer regions of YDV templates. Attempts were made to reduce the generation of these bands without sacrificing sensitivity; however, reducing the YanR primer concentration or diluting cDNA template beyond fourfold reduced sensitivity considerably. Malmstrom and Shu (2004) also reported additional bands in their YDV multiplex PCR reactions using YanR in the cDNA step.

As a confirmation of the specificity of the multiplex primers to discriminate PAV and PAS in single and dual infections, the general YDV primers ShuF and YanR (Malmstrom and Shu 2004) were used to amplify an $\sim 830$-bp region of ORF3/ORF4 (CP/MP) followed by single restriction digest of the resulting amplicon in a manner previously described (Kundu et al. 2009). After confirmation of single amplicons by electrophoresis, $10 \mu$ l of amplicon was digested with $H p a I I$ in a $15-\mu l$ reaction mix containing $1.5 \mu l$ of 10× NEB CutSmart buffer and 2.5 units of HpaII (New England BioLabs, Ipswich, MA), incubating the reaction for $18 \mathrm{~h}$ at $37^{\circ} \mathrm{C}$. In both single and mock dual mixtures, digested PAS amplicons yielded fragment sizes of 391, 325, 71, and $44 \mathrm{bp}$, whereas digested PAV samples yielded fragment sizes of 369, 259, 132, and 71 bp (Fig. 2C). In addition, digestion patterns of CP/MP amplicons derived from MAV or SGV single-infection positive controls further provided a means of confirming the identities of YDV species determined by the new multiplex method.

\section{The New Multiplex Assay Identified Six YDVs in Wheat Field Samples and Highlighted the Prevalence of PAS}

The utility of the multiplex RT-PCR assay was determined by testing the protocol with a subset of archived symptomatic singletiller Kansas field samples with known ELISA status (Rotenberg et al. 2016) and a few samples collected from two breeding nurseries in Riley County, KS. The subset consisted of 89 samples ( $\sim 12 \%$ of the entire collection of symptomatic samples) representing all nine wheat-growing regions of the state, 27 counties in total, from commercial fields and Kansas Agriculture Experiment Station variety trial sites. The breakdown of these samples by ELISA status was as follows: 48 displayed typical virus-like symptoms but were determined to be ELISA negative for PAV, RPV, Soil-borne wheat mosaic virus, Wheat spindle streak mosaic virus, Wheat streak mosaic virus (WSMV), and High plains wheat mosaic virus; 41 
were symptomatic and ELISA positive for at least one of the species, predominantly PAV and WSMV. The breeding nursery set of samples consisted of seven single-plant samples collected in 2015 and 2016, and this set was included solely to test the assay on freshly available tissue. The full set of cDNA primers (three in total, including the ubiquitin reference primer) and PCR primers (seven in total, including the ubiquitin reference primers) were used as described above for the multiplex assay on the mock full group of six YDV species. The multiplex assay on the field samples resulted in amplicons of the expected size consistent with the mock mixtures (Fig. 2B depicts a representative subset). At least one amplicon identifying each of six species was cloned and sequenced to verify the amplified target, which in all cases confirmed the target species. As demonstrated by the banding patterns of single and dual mixtures of positive controls (Fig. 2C), all archived samples were confirmed by HpaII digestion with consistent results (data not shown).

The YDV species occurrence in single or mixed infection in the 89 archived BYD symptomatic tissue samples and seven fresh field samples is reported in Table 3. Of the ELISA-positive archived samples, 93\% (38/41) were positive for at least one of the six YDVs in the multiplex panel. Of the ELISA-negative samples, 81\% (41/48) were positive for YDVs, indicating enhanced sensitivity of PCR compared with the ELISA method for detection. The archived samples and the fresh tiller samples (7/8 infected) were enriched for the KS major group of predominant species (PAS, PAV, and RPV) in single or mixed infections (Table 3, Fig. 2B). Over both types of samples, PAV $(63 \%, 60 / 96)$ and PAS $(57 \%, 55 / 96)$ were the most common species, and coinfection with these two commonly occurred (31\%, 30/96; Table 3, Fig. 2B). Incidentally, 16 samples that tested positive for PAV by ELISA in the published survey were determined to be single infections of PAS by the multiplex and restriction fragment length polymorphism assays in the present study, an indication that the Agdia commercial polyclonal antibody for the $\mathrm{CP}$ of PAV cross-reacts with the PAS CP (Chay et al. 1996). This finding could indicate that the occurrence of PAV may have been previously overrepresented in diagnostic reports coming out of the Great Plains and other regions of the United States, because this commercial kit is used routinely (Burrows et al. 2009; Hadi et al. 2012). The other four YDV species were detected exclusively in coinfection with PAS and/ or PAV, for which RPV $(16 \%, 15 / 96)$ and SGV $(9 \%, 8 / 89)$ were the most common of the four, followed by MAV $(5 \%, 5 / 96)$ and RMV-MT $(2 \%, 2 / 96)$. Fisher exact tests of independence revealed significant associations between YDV species occurrence (as determined by RT-PCR here) and ELISA status of the symptomatic tillers, whereby PAS and RPV were more frequently associated with ELISA-positive tillers, and PAV and SGV were more frequently associated with ELISA-negative tillers (two-sided $P<0.005$ ).

A small number of asymptomatic tillers were also examined by the multiplex RT-PCR assay ( $n=10$, data not shown). Nine were ELISA-positive, all of which were positive for at least one of the six YDVs. Of these samples, PAS was detected in $89 \%$ of the samples $(n=8)$ and PAV in 33\% $(n=3)$; mixed PAS/PAV infections were found in $22 \%(n=2)$ of these samples. The one ELISA-negative sample was PCR negative in the panel.

One significant finding from this work was the widespread detection of PAS in Kansas winter wheat fields. PAS and PAV were equally prevalent in the set of symptomatic tillers tested here, and PAS tended to be associated with ELISA-positive samples, whereas PAV tended to be associated with ELISA-negative samples $(P<0.005)$. Additional studies are planned to examine this phenomenon more fully and to empirically determine if Kansas isolates of PAS and PAV differ in their accumulation in plants or symptom expression. Others have previously reported the prevalence of PAV compared with the minor occurrence of PAS in wheat fields in South Carolina (Gray et al. 1998) and New York (Hall et al. 2010), and one controlled set of experiments reported that in mixed infections, PAV accumulated to higher amounts in wheat plants and had a higher likelihood of aphid transmission than PAS (Hall and Little 2013). In the Czech Republic, a survey reported that although PAS was more common than PAV in grasses and cereals, including winter wheat, field experimentation revealed that PAV caused more severe symptoms-reduced plant height and grain yield — on susceptible winter wheat and barley lines, posing a greater threat than PAS (Jarošová et al. 2013). Given the results of these previous studies, the large number of samples infected with PAS in Kansas is intriguing. The prevalence of this YDV species in consecutive seasons may point to the genetic variation in PAS populations under the intensive wheat-cropping system in Kansas. Several viral genome regions, from the RTP to the $3^{\prime}$ end of the genome, showed significant variation compared with publicly available PAS reference sequences. For example, P6 of the Kansas YDV isolates encoded by ORF6 varied considerably from the reference isolates for each species and from each other. Interestingly, P6 was characterized molecularly to function as a silencing suppressor for GAV (Liu et al. 2012), a YDV species reported in China. The role that silencing suppressors play in viral RNA accumulation has been documented with Cucumber mosaic virus (Du et al. 2008), in which

\begin{tabular}{|c|c|c|c|c|c|}
\hline \multirow[b]{3}{*}{ YDV species } & \multirow{2}{*}{\multicolumn{2}{|c|}{ Archived }} & \multirow{2}{*}{\multicolumn{2}{|c|}{ Fresh }} & \multirow[b]{3}{*}{ Total $(n=96)$} \\
\hline & & & & & \\
\hline & ELISA positive $(n=41)$ & ELISA negative $(n=48)$ & Symptoms $(n=5)$ & No symptoms $(n=2)$ & \\
\hline BYDV-PAS & 16 & 8 & 1 & 0 & 25 \\
\hline BYDV-PAS/PAV mixed ${ }^{c}$ & 15 & 13 & 2 & 0 & 30 \\
\hline BYDV-SGV ${ }^{\mathrm{d}}$ & 1 & 7 & 0 & 0 & 8 \\
\hline BYDV-MAV & 1 & 4 & 0 & 0 & 5 \\
\hline MYDV-RMV, MT variant ${ }^{\mathrm{d}}$ & 1 & 1 & 0 & 0 & 2 \\
\hline No YDV detected & 3 & 7 & 0 & 1 & 11 \\
\hline
\end{tabular}

${ }^{a}$ ELISA status of samples determined previously using a test panel of six wheat viruses (Rotenberg et al. 2016).

${ }^{\mathrm{b}}$ Samples collected from symptomatic or asymptomatic hard red winter wheat in 2015 and 2016 from Kansas wheat breeding plots.

${ }^{\mathrm{c}}$ In single or mixed infection with other YDVs.

${ }^{\mathrm{d}}$ Found in mixed infection with PAS, PAV, or both. 
genetic variants of the silencing suppressor $(2 \mathrm{~b})$ protein resulted in varying levels of virus accumulation. Genetic variation in P6 of the KS PAS haplotypes may have a bearing on the accumulation of the virus in plant tissues and availability of virus for aphid acquisition, but this remains to be tested empirically. Biological and functional characterizations of these isolates are currently under way.

\section{Conclusions}

The new multiplex assay proved to be a useful technique in documenting single and mixed infections of YDVs in fields of an intensive wheat-growing region of the United States. We documented the widespread occurrence of PAS infecting commercial wheat cultivars throughout Kansas. Documenting the prevalence of PAS and genetic variants of YDV populations in other states in the Great Plains region will be important for developing effective regional virus-control strategies. The outcomes of this study underscore the challenges of keeping nucleic-acid-based diagnostic tools current to achieve robust detection of evolving plant virus populations of agricultural importance.

\section{Acknowledgments}

The authors thank Stewart Gray for providing positive controls for four of the viruses used in this study and for helpful discussions on developing the assay, Jesse Poland for planting and allowing access to wheat breeding nursery plots that served as the sites for obtaining fresh samples, and Anna Whitfield for critical review of the manuscript. National Center for Biotechnology (NCBI) GenBank genome sequence accessions to be released upon publication: KY593456 (KS PAS-1), KY593457 (KS PAS-2), and KY593458 (KS PAV).

\section{Literature Cited}

Acosta-Leal, R., Hervey, K., Whitfield, A. E., Bockus, W. W., and Rotenberg, D. 2013. Genetic diversity of Barley yellow dwarf virus (BYDV) populations in Kansas wheat fields. Phytopathology 103(Suppl. 3):S3.1.

Altschul, S. F., Gish, W., Miller, W., Myers, E. W., and Lipman, D. J. 1990. Basic local alignment search tool. J. Mol. Biol. 215:403-410.

Appel, J., De Wolf, E. D., Todd, T., and Bockus, W. W. 2015. Preliminary 2015 Kansas wheat disease loss estimates. Kansas Cooperative Plant Disease Survey Report. Kansas Department of Agriculture, Manhattan, KS.

Burrows, M., Franc, G., Rush, C., Blunt, T., Ito, D., Kinzer, K., Olson, J., O'Mara, J., Price, J., Tande, C., Ziems, A., and Stack, J. 2009. Occurrence of viruses in wheat in the Great Plains region, 2008. Plant Health Prog. Online publication. doi.org/10.1094/PHP-2009-0706-01-RS

Chay, C., Smith, D., Vaughan, R., and Gray, S. 1996. Diversity among isolates within the PAV serotype of Barley yellow dwarf virus. Phytopathology 86:370-377.

D'Arcy, C. J., and Domier, L. L. 2000. Barley yellow dwarf. Plant Health Instructor.

Deb, M., and Anderson, J. M. 2008. Development of a multiplexed PCR detection method for Barley and Cereal yellow dwarf viruses, Wheat spindle streak virus, Wheat streak mosaic virus and Soil-borne wheat mosaic virus. J. Virol. Methods 148:17-24.

Dieffenbach, C. W., Lowe, T. M. J., and Dveksler, G. S. 1993. General concepts for PCR primer design. Genome Res. 3:S30-S37.

Domier, L. L. 2012. Luteoviridae. Pages 1045-1053 in: Virus Taxonomy: Classification and Nomenclature of Viruses. Ninth Report of the International Committee on Taxonomy of Viruses. A. M. Q. King, E. Lefkowitz, M. J. Adams, and E. B. Carstens, eds. Academic Press, Cambridge, MA.

Du, Z., Chen, F., Zhao, Z., Liao, Q., Palukaitis, P., and Chen, J. 2008. The 2b protein and the $\mathrm{C}$-terminus of the $2 \mathrm{a}$ protein of Cucumber mosaic virus subgroup I strains both play a role in viral RNA accumulation and induction of symptoms. Virology 380:363-370.

Garrett, K. A., Dendy, S. P., Power, A. G., Blaisdell, G. K., Alexander, H. M., and McCarron, J. K. 2004. Barley yellow dwarf disease in natural populations of dominant tallgrass prairie species in Kansas. Plant Dis. 88:574.

Geske, S. M., French, R., Robertson, N. L., and Carroll, T. W. 1996. Purification and coat protein gene sequence of a Montana RMV-like isolate of Barley yellow dwarf virus. Arch. Virol. 141:541-556.
Gray, S. M., Chapin, J. W., Smith, D. M., Banerjee, N., and Thomas, J. S. 1998. Barley yellow dwarf luteoviruses and their predominant aphid vectors in winter wheat grown in South Carolina. Plant Dis. 82:1328-1333.

Hadi, B. A. R., Flanders, K. L., Bowen, K. L., Murphy, J. F., and Blount, A. R. 2012. Survey of Barley yellow dwarf virus and Cereal yellow dwarf virus on three perennial pasture grasses in Florida. J. Entomol. Sci. 47:35-43.

Hall, G. S., and Little, D. P. 2013. Within-host competition between barley yellow dwarf-PAV and -PAS. Virus Res. 174:148-151.

Hall, G. S., Peters, J. S., Little, D. P., and Power, A. G. 2010. Plant community diversity influences vector behaviour and Barley yellow dwarf virus population structure. Plant Pathol. 59:1152-1158.

Huang, X., and Madan, A. 1999. CAP3: A DNA sequence assembly program. Genome Res. 9:868-877.

Jarošová, J., Chrpová, J., Šíp, V., and Kundu, J. K. 2013. A comparative study of the Barley yellow dwarf virus species PAV and PAS: Distribution, accumulation and host resistance. Plant Pathol. 62:436-443.

Jin, Z., Wang, X., Chang, S., and Zhou, G. 2004. The complete nucleotide sequence and its organization of the genome of Barley yellow dwarf virusGAV. Sci. China Ser. C Life Sci. 47:175-182.

Kansas Department of Agriculture. 2013. Kansas Farm Facts. https://agriculture. ks.gov/docs/default-source/documents-office-of-the-secretary/kansas-farmfacts-august-2013.pdf?sfvrsn=5. Accessed April 21, 2016.

Krueger, E. N., Beckett, R. J., Gray, S. M., and Miller, W. A. 2013. The complete nucleotide sequence of the genome of Barley yellow dwarf virus-RMV reveals it to be a new Polerovirus distantly related to other yellow dwarf viruses. Front. Microbiol. 4:205.

Kundu, J. K., Jarošová, J., Gadiou, S., and Cervená, G. 2009. Discrimination of three BYDV species by one-step RT-PCR-RFLP and sequence based methods in cereal plants from the Czech Republic. Cereal Res. Commun. 37:541-550.

Liu, F., Wang, X., Liu, Y., Xie, J., Gray, S. M., Zhou, G., and Gao, B. 2007. A Chinese isolate of Barley yellow dwarf virus-PAV represents a third distinct species within the PAV serotype. Arch. Virol. 152:1365-1373.

Liu, Y., Zhai, H., Zhao, K., Wu, B., and Wang, X. 2012. Two suppressors of RNA silencing encoded by cereal-infecting members of the family Luteoviridae. J. Gen. Virol. 93:1825-1830.

Malmstrom, C. M., and Shu, R. 2004. Multiplexed RT-PCR for streamlined detection and separation of barley and cereal yellow dwarf viruses. J. Virol. Methods 120:69-78.

Mayo, M. A. 2002. Virus taxonomy-Houston 2002. Arch. Virol. 147:1071-1076.

Miller, W. A., Waterhouse, P. M., and Gerlach, W. L. 1988. Sequence and organization of Barley yellow dwarf virus genomic RNA. Nucleic Acids Res. 16:6097-6111.

Paolacci, A. R., Tanzarella, O. A., Porceddu, E., and Ciaffi, M. 2009. Identification and validation of reference genes for quantitative RT-PCR normalization in wheat. BMC Mol. Biol. 10:11.

Rochow, W. F., and Muller, I. 1971. A fifth variant of Barley yellow dwarf virus in New York. Plant Dis. Rep. 55:874-877.

Rombel, I. T., Sykes, K. F., Rayner, S., and Johnston, S. A. 2002. ORFFINDER: A vector for high-throughput gene identification. Gene 282:33-41.

Rotenberg, D., Bockus, W. W., Whitfield, A. E., Hervey, K., Baker, K. D., Ou, Z., Laney, A. G., De Wolf, E. D., and Appel, J. A. 2016. Occurrence of viruses and associated grain yields of paired symptomatic and nonsymptomatic tillers in Kansas winter wheat fields. Phytopathology 106:202-210.

Sievers, F., Wilm, A., Dineen, D., Gibson, T. J., Karplus, K., Li, W., Lopez, R., McWilliam, H., Remmert, M., Soding, J., Thompson, J. D., and Higgins, D. G. 2014. Fast, scalable generation of high-quality protein multiple sequence alignments using Clustal Omega. Mol. Syst. Biol. 7:539.

Svanella-Dumas, L., Candresse, T., Hullé, M., and Marais, A. 2013. Distribution of Barley yellow dwarf virus-PAV in the sub-Antarctic Kerguelen Islands and characterization of two new Luteovirus species. PLoS One 8: e67231.

Ueng, P. P., Vincent, J. R., Kawata, E. E., Lei, C.-H., Lister, R. M., and Larkins, B. A. 1992. Nucleotide sequence analysis of the genomes of the MAV-PS1 and P-PAV isolates of Barley yellow dwarf virus. J. Gen. Virol. 73: 487-492.

Vincent, J. R., Lister, R. M., and Larkins, B. A. 1991. Nucleotide sequence analysis and genomic organization of the NY-RPV isolate of Barley yellow dwarf virus. J. Gen. Virol. 72:2347-2355.

Wu, D. Y., Ugozzoli, L., Pal, B. K., and Wallace, R. B. 1989. Allele-specific enzymatic amplification of beta-globin genomic DNA for diagnosis of sickle cell anemia. Proc. Natl. Acad. Sci. U.S.A. 86:2757-2760.

Zhang, W., Cheng, Z., Xu, L., Wu, M., Waterhouse, P., Zhou, G., and Li, S. 2009. The complete nucleotide sequence of the barley yellow dwarf GPV isolate from China shows that it is a new member of the genus Polerovirus. Arch. Virol. 154:1125-1128. 